Polymer Journal, Vol. 10, No. 3, pp 341-351 (1978)

\title{
Application of Light Scattering from Dielectric Cylinder Based upon Mie and Rayleigh-Gans-Born Theories to Polymer Systems. I. Scattering from a Glass Fiber
}

\author{
Yukikazu Uemura, ${ }^{*}$ Mineo Fujimura, Takeji Hashimoto, \\ and Hiromichi KaWAI \\ Department of Polymer Chemistry, Faculty of Engineering, \\ Kyoto University, Kyoto 606, Japan.
}

(Received December 9, 1977)

\begin{abstract}
Light scattering from a glass fiber was investigated for a rigorous evaluation of its size and refractive index. Angular variation of forward scattering intensity from the fiber of about $5 \mu \mathrm{m}$ in radius with or without immersion liquid was quantitatively measured by a laser photometric apparatus. The results were analyzed by Mie and Rayleigh-Gans-Born (R.G.B.) scattering from an isolated and infinitely long cylinder. Discussions were made on criterion of R.G.B. scattering, when the theory is applied to the system, and on approximated but rapid and convenient methods in evaluating the size and refractive index of the fiber.

KEY WORDS Mie Scattering / Rayleigh-Gans-Born Scattering /

E-Glass Fiber / Cylinder / Size / Refractive Index /
\end{abstract}

Light scattering from most of the polymeric systems has been described in terms of the theories of Rayleigh, ${ }^{1}$ Gans, ${ }^{2}$ and Born $^{3}$ (R.G.B. theory) which are based upon two basic assumptions as described in detail by Van de Hulst: ${ }^{4}$

$$
\begin{aligned}
& \text { (a) }\left|m_{\mathrm{r}}-1\right| \ll 1 \\
& \text { (b) } 2 k_{0} a\left|m_{\mathrm{r}}-1\right| \ll 1
\end{aligned}
$$

where $m_{\mathrm{r}}=m_{1} / m_{3}$, the ratio of refractive indices of the particle of radius $a$ (when the particle is spherical) and its surrounding medium, respectively, $k_{0}=2 \pi / \lambda_{0}$, and $\lambda_{0}$ is the wavelength of light in vacuo. The assumptions are strictly valid for X-ray scattering, and are generally valid with good accuracy for scattering from polymer solutions and from crystalline and amorphous solids in which the refractive index differences between the particles and surrounding media are usually so small that eq 1 is always satisfied.

In this case the scattering electric vectors $E_{\mathrm{s}}$ at far field is simply given by the sum of the

\footnotetext{
* Present Address: Sumitomo Chemical Co. Ltd., 1-98, Kasugade-Naka 3-chome, Konohana-ku, Osaka, 554, Japan.
}

scattering field $E_{j}$ from the $j$-th volume elemen $\mathrm{t}$ in the particle which in turn is given as a solution of Maxwell equation of radiation of electromagnetic wave from oscillating dipole, ${ }^{4,5}$

$$
\begin{aligned}
& E_{\mathrm{s}}=\sum_{j} E_{j} \\
& E_{j}=\left(k_{0}{ }^{2} / R\right)\left(\boldsymbol{M}_{j} \cdot \mathbf{O}\right) \exp \left(i \boldsymbol{h} \cdot \boldsymbol{r}_{j}\right)
\end{aligned}
$$

where $R$ is the distance between the scattering center and point of observation, $\left(\boldsymbol{M}_{j} \cdot \mathbf{O}\right)$ the effective induced dipole moment of the $j$ th scattering element where $\mathbf{O}$ is the unit vector parallel to the polarization direction of the analyzer placed in between the specimen and the detector for the measurements of depolarized components of the scattered light. The exponential term is a phase factor of the $j$ th element in the R.G.B. theory as will be described later. The R.G.B. scattering is widely applied to characterize heterogeneities in polymer solutions ${ }^{6}$ and solids. ${ }^{7}$

When the refractive index difference becomes greater such as in the case of colloidal dispersion of sulfur in water, ${ }^{8}$ polymer lattices in emulsion, ${ }^{9}$ and glass fibers, beads and voids in polymer matrices (composite mateterials in general), ${ }^{10}$ the 
R.G.B. theory is not valid and the scattering phenomena from such systems becomes more complex due to the perturbations of the scattered and incident electric fields within the scattering medium which, in turn, result from the electromagnetic interaction between the incident field and polarized scattering elements. ${ }^{4,10,11}$

Rigorous solutions of scattering from such complex but general systems-Mie scatteringhave been obtained for relatively limited systems of simple scattering geometries such as sphere, coated sphere, simple or coaxial cylinder, ellipsoid of revolution, and etc.,10 The Mie scattering from an isolated sphere is expressed by the Mie series, i.e., the series of products of Legendre polynomials and Bessel functions, the expression being much more complicated than the R.G.B. equation. However, the Mie scattering theory is more general than the R.G.B. theory and is applicable to more general systems of practical importance.

In this series of article, we will discuss an application of Mie scattering from a simple cylinder or a coaxial cylinder to that from grass fibers and from polymer composites. The Mie scattering from the cylinder systems has been developed by Churchill ${ }^{12)}$ for dielectric or conducting cylinders for normal incidence, and by Kerker, et al.,${ }^{13}$ and Farone, et al. ${ }^{14}$ for oblique incidence. The application of the theory to the submicron cylinder has been worked out by Kerker, et al., ${ }^{15}$ and by Lundberg. ${ }^{16}$ Rigorous solution of scattered electromagnetic wave has been obtained also for coaxial cylinder by Tang, ${ }^{17}$ Evans, et al. ${ }^{18}$ and Kerker, et al. ${ }^{19}$ Yeh has extended the theory to the cylider with an elliptical cross section. ${ }^{20}$ In all these cases the cylinder has been treated as infinitely long and homogeneous.

The scattering from the coaxial cylinder may be applied to the scattering from glass fibers coated by the coupling agents and/or embedded in the polymer matrix, and also to the scattering from glass fiber reinforced plastics (FRP) with void or density-lowered phase at the fiber-matrix interface which may be generated by application of stress. Such application will be described in subsequent papers. ${ }^{23}$

In this article, we will discuss the application of Mie scattering to an $E$-glass fiber ${ }^{21}$ of radius of about $5 \mu \mathrm{m}$, to estimate its size and refractive index. The fiber is long enough to be treated as an infinitely long cylinder, and has a radius much larger than the wavelength of an incident light beam $\left(\lambda_{0}=6328 \AA\right.$, He-Ne gas laser). Our primary interest is focussed on the angular variation of the scattered intensity in forward direction. To our knowledge, the angular variation of the forward scattering from such large fiber has not often been treated so far. ${ }^{16}$

\section{THEORIES}

\section{R.G.B. Scattering by an Infinitely Long Cylinder}

Let us first consider the R.G.B. scattering from an isolated and infinitely long cylinder of radius $R_{1}$ and refractive index $m_{1}$ embedded in isotropic and homogeneous medium of refractive index $m_{3}$. The cylinder which is assumed to be isotropic and homogeneous (good assumption for the $E$-glass fiber) is perfectly oriented along $z$-axis as shown in Figure 1. We are only interested

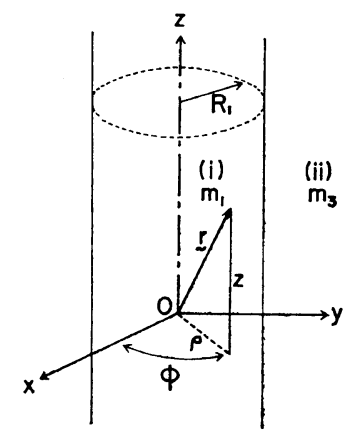

Figure 1. Coordinate system of a glass fiber. The axis is parallel to $0 z$.

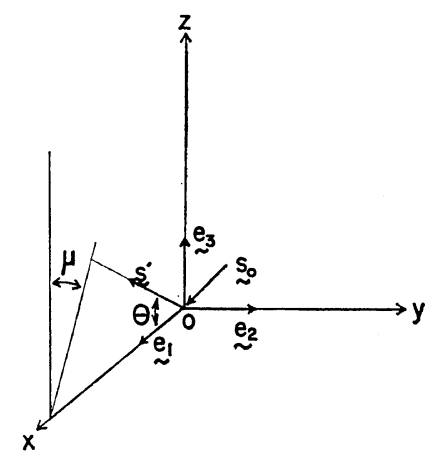

Figure 2. Definition of scattering angle $\theta$, azimuthal angle $\mu$, and unit vectors $e_{1}, e_{2}$, and $e_{3}$. 
in the equatorial scattering, i.e., the scattering in the plane $X O Y$ for which the azimuthal angle $\mu$ is 90 degrees as a function of the scattering angle $\theta$ (see Figure 2).

The $V_{v}$ scattering amplitude at far field $E_{V_{v}}$ in which scattering is observed for vertically polarized incident field and through vertically polarized analyzer is given by ${ }^{4,10}$

$$
E_{V_{\mathrm{v}}}(\theta)=\left(k_{0}{ }^{2} / R\right) \int(\boldsymbol{M} \cdot \mathbf{O})_{V_{\mathrm{v}}} \exp (i \boldsymbol{h} \cdot \boldsymbol{r}) \mathrm{d} \boldsymbol{r}
$$

where $\boldsymbol{M}=\alpha(\boldsymbol{r}) E_{0} \boldsymbol{e}_{3}, \mathbf{O}=\boldsymbol{e}_{3}, \quad \alpha(\boldsymbol{r})$ is the polarizability at point $r$, and $e_{3}$ is the unit vector along the $z$-axis. The cylinder is assumed to be optically isotropic and homogeneous, so that $\alpha(\boldsymbol{r})=$ $\alpha_{0}=$ (const.)

$$
(\boldsymbol{M} \cdot \mathbf{O})_{V_{\mathrm{v}}}=\alpha_{0} E_{0}
$$

where $E_{0}$ is the electric field of the incident beam. Using the cylindrical coordinate shown in Figure 1, it follows that

and

$$
\boldsymbol{r}=\rho\left(\boldsymbol{e}_{1} \cos \phi+\boldsymbol{e}_{2} \sin \phi\right)+z \boldsymbol{e}_{3}
$$

$$
\begin{aligned}
\boldsymbol{h} & =k\left(\boldsymbol{s}^{\prime}-\boldsymbol{s}_{0}\right) \\
& =k\left[(1-\cos \theta) \boldsymbol{e}_{1}-(\sin \theta) \boldsymbol{e}_{2}\right],
\end{aligned}
$$

where $e_{1}$ and $e_{2}$ are the unit vectors along the $x$ - and $y$-axes, respectively, $s^{\prime}$ and $s_{0}$ are the unit vectors along the propagation directions of the scattered and incident rays, respectively, $\lambda$ is the wavelength of light in the medium, and $k$ is given by $(2 \pi / \lambda)$ (see Figure 2).

From eq 3 to 6 , it follows that

$$
E_{V_{\mathrm{v}}}(\theta)=\text { (const.) } \int_{0}^{R_{1}} m(\rho) J_{0}[2 k \rho \sin (\theta / 2)] \rho \mathrm{d} \rho
$$

where the local refractive index $m(\rho)$ is $m_{1}$ within the cylinder and $m_{3}$ outside the cylinder, $J_{0}$ is the Bessel function of order zero, and the constant is related to the absolute intensity. After integration, we obtain an expression for the $V_{\mathrm{v}}$ scattering intensity as follows:

$$
\left.I_{V_{\mathrm{v}}}(\theta)=\text { (const. }\right)\left(m_{1}-m_{3}\right)^{2}\left[J_{1}\left(U_{1}\right) / U_{1}\right]^{2}
$$

where $J_{1}$ is the Bessel function of the first order, and

$$
U_{1}=(4 \pi / \lambda) R_{1} \sin (\theta / 2)
$$

Mie Scattering by an Infinitely Long Cylinder

Let us consider Mie scattering for the same cylinder under the same conditions as above. The solution of the Maxwell equation for the scattered electromagnetic field must be obtained under proper boundary conditions. ${ }^{10)}$ If $\xi$ is the solution of the scalar wave equation satisfying the Maxwell equations, $\xi$ in each region of Figure 1 for vertically polarized incident beam is given by

$$
\begin{gathered}
\xi=\sum_{n=-\infty}^{\infty} F_{n} B_{n}{ }^{\prime} J_{n}\left(m_{1} k \rho\right), \text { for region (i) } \\
\xi=\sum_{n=-\infty}^{\infty} F_{n}\left[J_{n}\left(m_{3} k \rho\right)-b_{n} H_{n}\left(m_{3} k \rho\right)\right] \\
\text { for region (ii) }
\end{gathered}
$$

where

$$
F_{n}=(-1)^{n} \exp [i(n \theta+\omega t)]
$$

and $\omega$ is the angular frequency of the incident field. The functions $J_{n}$ and $H_{n}$ are the $n$th order Bessel function of the first kind and Hankel function of the second kind, respectively. The constants $B_{n}{ }^{\prime}$ and $b_{n}$ are determined by the boundary conditions for the electromagnetic field that $m \xi$ and $m(\partial \xi / \partial \rho)$ ( $m$ being the refractive index) should be cotinuous at the boundary.

The constant $b_{n}$ in the region (ii) is associated with the scattered intensity at far field. If $\alpha$ is defined by

$$
\alpha=k_{0} R_{1}
$$

then $b_{n}$ is given by

$$
b_{n}=\frac{m_{1} J_{n}\left(m_{3} \alpha\right) J_{n}{ }^{\prime}\left(m_{1} \alpha\right)-m_{3} J_{n}{ }^{\prime}\left(m_{3} \alpha\right) J_{n}\left(m_{1} \alpha\right)}{m_{1} H_{n}\left(m_{3} \alpha\right) J_{n}{ }^{\prime}\left(m_{1} \alpha\right)-m_{3} H_{n}{ }^{\prime}\left(m_{3} \alpha\right) J_{n}\left(m_{1} \alpha\right)}
$$

where

$$
\begin{aligned}
J_{n}{ }^{\prime}\left(m_{1} \alpha\right) & =\left[\mathrm{d} J_{n}(x) / \mathrm{d} x\right]_{x=m_{1} \alpha} \\
H_{n}{ }^{\prime}\left(m_{3} \alpha\right) & =\left[\mathrm{d} H_{n}(x) / \mathrm{d} x\right]_{x=m_{3} \alpha}, \quad \text { and etc. }
\end{aligned}
$$

At the point of observation where $\rho=R$, far from the scattering center, the Hankel function in eq 11 is approximated by

$$
\begin{aligned}
H_{n}\left(m_{3} k_{0} R\right) & \simeq\left(\pi m_{3} k_{0} R / 2\right)^{-1 / 2} \\
& \times \exp \left\{-i\left[m_{3} k_{0} R-(2 n+1) \pi / 4\right]\right\}
\end{aligned}
$$

and the incident wave propagating along the $x$-axis is given by

$$
\xi_{0}=\exp \left[-i\left(m_{3} k_{0} x-\omega t\right)\right]
$$

Therefore, the scattered wave at far field is given by 


$$
\begin{gathered}
\xi=\left(\pi m_{3} k_{0} R / 2\right)^{-1 / 2} \exp \left\{-i\left[m_{3} k_{0} R-\omega t-(3 / 4) \pi\right]\right\} \\
\times \sum_{n=-\infty}^{\infty} b_{n} \exp (i n \theta)
\end{gathered}
$$

The differential scattering cross section $I_{V_{\mathrm{v}}}(\theta)$ at far field under the $V_{\mathrm{v}}$ polarization condition is given from eq 11 to 17 by

$$
\begin{aligned}
I_{V_{\mathrm{v}}}(\theta) / I_{0} & =\left(\pi^{2} R m_{3} / \lambda_{0}\right)^{-1} \mid\left.\sum_{n=-\infty}^{\infty} b_{n} \exp (\text { in } \theta)\right|^{2} \\
& =\left(\pi^{2} R m_{3} / \lambda_{0}\right)^{-1}\left|b_{0}+2 \sum_{n=1}^{\infty} b_{n} \cos n \theta\right|^{2}
\end{aligned}
$$

where $I_{0}$ is incident beam intensity.

Angular distribution of the scattered light can be numerically calculated from eq 13 and 18 , providing that $m_{1}, m_{3}$, and $R_{1}$ are all known. The scattering efficiency $Q$, the scattering cross section divided by the geometric cross section of the scattering object, is also obtained from eq 18 ,

$$
Q=(\lambda / \pi R)\left[\left|b_{0}\right|^{2}+2 \sum_{n=1}^{\infty}\left|b_{n}\right|^{2}\right]
$$

\section{EXPERIMENTS}

The E-glass monofilament, a long glass fiber, ${ }^{2}$ was used as a test specimen for light scattering experiment. The diameter of the glass fiber measured from an optical microscope is $10 \mu \mathrm{m}$ and refractive index is $1.548 .^{211}$ The fiber was immersed in silicone oils with various refractive indices $\left(m_{3}=1.560,1.528,1.516,1.504\right.$, and 1.498) to investigate its effect on scattering. The refractive indices of the oils were measured by the Abbe refractometer.

A He-Ne gas laser (15-mW power with a single mode, NAL-705 GS, Nippon Kagaku Inc., Osaka, Japan) was used as plane polalized incident beam. The angular dependence of scattered intensity was measured by scanning a photomultiplier tube in a horizontal plane. The analyzer was placed behind the specimen and in front of the photomultiplier tube. The $V_{\mathrm{v}}$ scattered intensity was measured by setting the polarization directions of incident beam, analyzer, and the axis of the glass fiber all vertically. The polarization direction of the incident beam was adjusted by a polarization rotator (Spectra Physics Inc., model 310).

\section{RESULTS AND DISCUSSIONS}

\section{Numerical Calculation}

The Mie scattering for an infinitely long cylinder was numerically calculated based upon eq 13 and 18. The value of the Bessel function of order $n$ and of arbitrary variable $x, J_{n}(x)$, was evaluated according to Miller's method, ${ }^{22}$ in which a small arbitrary value was given for $J_{n}(x)$ for the required maximum $n$ and then, $J_{n}(x)$ 's for the lower orders were calculated by using the recursion relationship of the Bessel function. Finally a series of true $J_{n}(x)$ was obtained by determining its proportional constant from the relationship:

$$
J_{0}(x)+2 \sum_{n=1}^{\infty} J_{2 n}(x)=1
$$

In the case of the Neumann function, $Y_{0}(x)$ for the zeroth order and for an arbitrary variable $x$ was calculated first, and then $Y_{n}(x)$ for the ascending orders were calculated by using the recursion relationship. ${ }^{24}$

The R.G.B. scattering from the cylinder was also numerically calculated based upon eq 8 and 9.

\section{Experimental Results}

Observation of the glass fiber under phase contrast optical micrograph indicated that the glass fiber is uniform, having a radius of $5.0 \mu \mathrm{m}$, the value of which will be confirmed by light scattering experiments, as will be discussed later. Figure 3 shows the $V_{\mathrm{v}}$ light scattering patterns of the glass fiber alone (case of $m_{3}=1.0$ ) or the glass fiber immersed in silicone oils of various refractive indices $\left(m_{3}=1.498\right.$ to 1.560$)$. The scattered intensity of the immersed fiber tends to decrease with increasing $\theta$ more rapidly than the intensity from the fiber alone. The scattered intensity is minimized for the silicone oil of refractive index of 1.560 which is the closest value to that of the glass fiber.

Figure 4 shows the measured equatorial scattering of the glass fiber as a function of scattering angle $\theta_{\mathrm{a}}$ in air (dotted line) together with the calculated scattering curve (Mie scattering) which closely fits the experimental curve as described later. Figure 5 typically represents the effects of immersing the fiber in the silicone oil on the scattered intensity distribution of the 
G.F. in

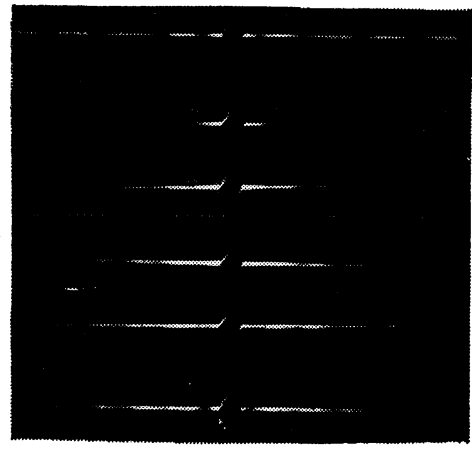

(a)

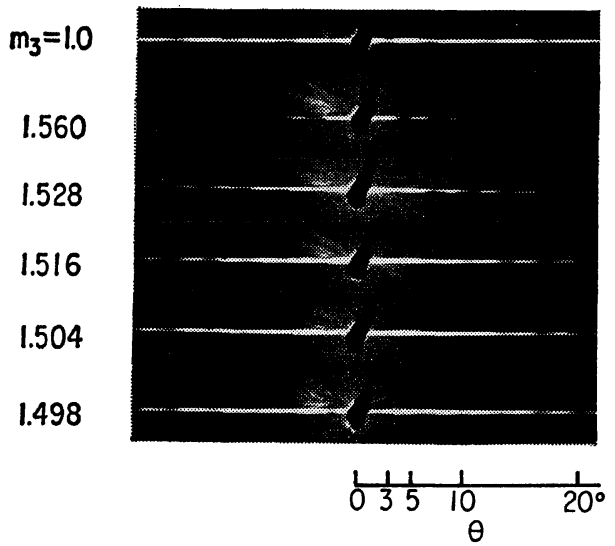

(b)

Figure 3. Photographic light scattering patterns from the E-glass fiber with two different exposure (a) and (b). $m_{3}$ is the refractive index of the surrounding medium.

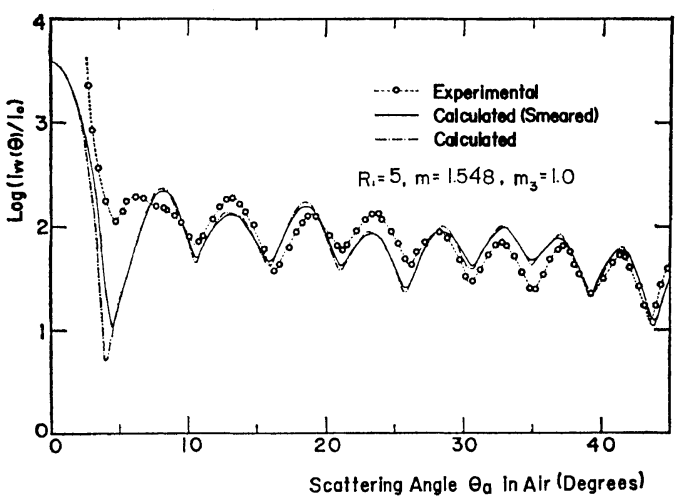

Figure 4. Equatorial relative scattering intensity distribution of the $E$-glass fiber. The dotted line is the experimental result The solid line and dash-dot lines are the smeared calculated and calculated curves, respectively, for $R_{1}=5.0 \mu \mathrm{m}$ and $m=1.548$.

glass fiber (dotted lines). The peak positions and their shifts by the immersion liquid coincide with those observed in the photographic patterns in Figure 3. It should be noted that the scattering angle in the figure is not the angle in air $\left(\theta_{\mathrm{a}}\right)$ but the one in the silicone oil of refractive index $m_{3}(\theta)$. The angle $\theta$ is estimated from the angle $\theta_{\mathrm{a}}$,

$$
\theta=\sin ^{-1}\left[\left(1 / m_{3}\right) \sin \theta_{\mathrm{a}}\right]
$$

The calculated results of Mie scattering are also shown in Figure 5 for a few values of $m_{3}$ and

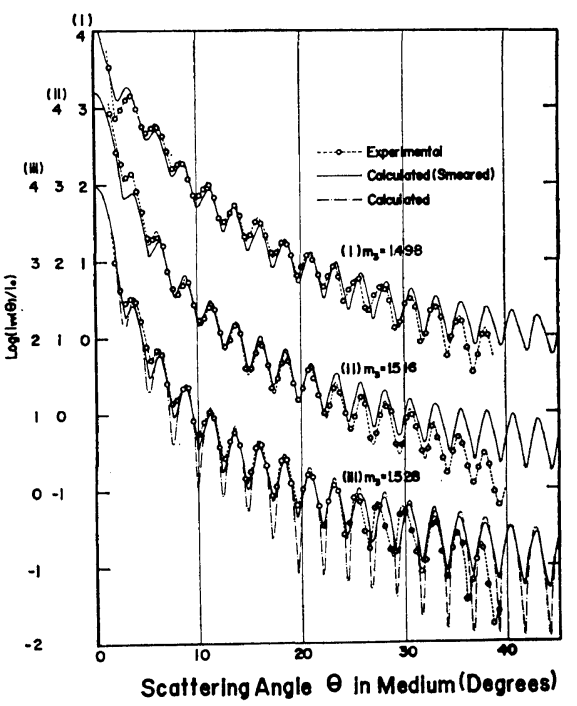

Figure 5. Equatorial relative scattering intensity distribution of the $E$-glass fiber embedded in the silicone oils of refractive indices, $m_{3}=1.498,1.516$, and 1.528. The dotted, solid, and dash-dot lines have the same meaning as above. $R_{1}=5.0 \mu \mathrm{m}$ and $m=1.548$.

for the fixed values of $R=5.0 \mu \mathrm{m}$ and $m_{1}=$ 1.548. This will be discussed later.

Upon immersing the glass fiber into the immersion liquid, it is seen that the maxima shift toward the small scattering angles and that the scattered intensity decreases with increasing $\theta$ more rapidly than that from the fiber alone. 
The shift of the scattering maxima may be qualitatively interpreted in terms of the refraction of scattered light at the interface between the glass fiber and silicone oil; i.e., the closer the refractive indices of the oil and the fiber, the smaller is the angle of refraction of the scattered beam at the interface, leading to the shift of the maximum intensity toward smaller scattering angles.

Calculated Results of Mie Scattering by an Infinitely Long Cylinder and Comparison with Experimental Scattering

In this section we will discuss Mie scattering from a fiber in air with $m_{1}=m$ and $m_{3}=1$. In this case eq 13 is given by

$$
\begin{aligned}
b_{n}= & {\left[m J_{n}(\alpha) J_{n}{ }^{\prime}(m \alpha)-J_{n}{ }^{\prime}(\alpha) J_{n}(m \alpha)\right] /\left[m H_{n}(\alpha) J_{n}{ }^{\prime}(m \alpha)\right.} \\
& \left.-H_{n}{ }^{\prime}(\alpha) J_{n}(m \alpha)\right]
\end{aligned}
$$

In all calculations $\lambda_{0}$ is set to $6328 \AA$, the wavelength of incident beam from a He-Ne gas laser. It is obvious from eq 18 and 21 that the relative angular dependence of $I_{V_{\mathrm{V}}}$ with scattering angles depends upon two independent parameters of $m$ and $R_{1}$. In contrast to the Mie scattering, the R.G.B. scattering from the system is given from eq 8 and 9 . Therefore the relative angular dependence of the R.G.B. scattering depends only upon the reduced variable $U_{1}$ or upon the product of $m$ and $R_{1}$.

Figure 6 shows the effect of radius $R_{1}$ on the angular dependence of Mie scattering where $m$ is assumed to be 1.548. Upon increasing the radius $R_{1}$, the scattering maxima generally shift toward smaller angles and the angular separa-

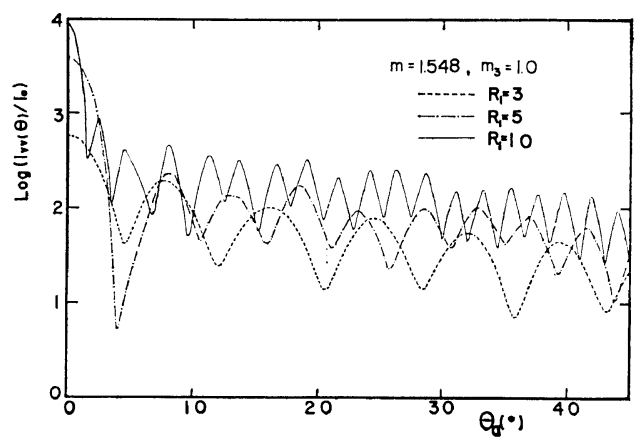

Figure 6. Calculated equatorial scattering curves for a cylinder of $m=1.548$ as a function of radius $R_{1} . \quad m_{3}=1.000$.

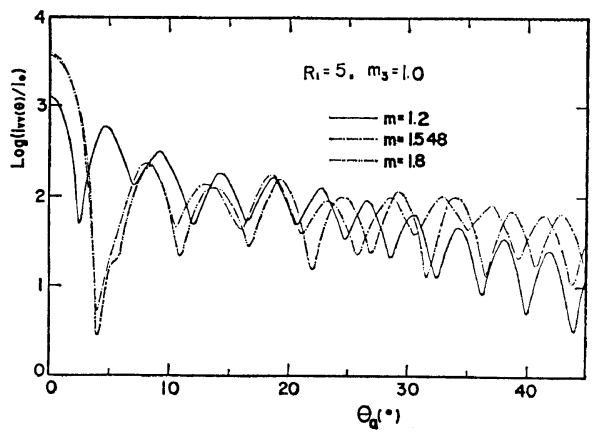

Figure 7. Calculated equatorial scattering curves for a cylinder of $R_{1}=5.0 \mu \mathrm{m}$ as a function of $m$. $m_{3}$ is kept at 1.000 .

tion between the successive maxima decreases. The angular separation systematically decreases to become a constant value with an increase in the scattering angles as is clearly seen in Figure 11.

Figure 7 shows effect of the refractive index $m$ of the fiber on angular dependence of Mie scattering for $R_{1}=5.0 \mu \mathrm{m}$. By decreasing the refractive index of the fiber, the difference of the refractive indices between the fiber and the medium decreases, so that the scattering maxima shift toward smaller angles, and therefore the number of scattering maxima within a given angular interval increases. This may be qualitatively interpreted on the basis of refraction of the scattered light at the fiber matrix-interface; i.e., the smaller the value of $m$, the smaller the difference of the refractive indices between the fiber and the medium, and so consequently the shifts of the scattered rays due to the refraction toward larger scattering angles become small. As seen from the effect of radius $R_{1}$ on the scattering curves, the angular separation between the successive scattering maxima decrease to a constant value with an increase in the scattering angle. It is also seen that with decreasing $m$, maximum scattered intensity tends to decrease rapidly with increasing the orders of the maxima in a manner similar to the R.G.B. scattering (see Figure 9).

Figure 4 shows the calculated Mie scattering intensity distribution for $R_{1}=5.0 \mu \mathrm{m}, m=1.548$, and $m_{3}=1.0$ and for $\lambda_{0}=6328 \AA$ (wavelength of a $\mathrm{He}-\mathrm{Ne}$ gas laser beam). Direct comparison of the calculated and experimental scattering curves has yielded a possible set of $m(=1.548)$ 
and $R_{1}(=5 \mu \mathrm{m})$, which is quite close to those reported for the $E$-glass. ${ }^{22}$ A better curve fit will be obtained by finer adjustments of the parameters.

It should be noted that the calculated scattering curves shown by dash-dot curves in Figures 4 and 5 correspond to the case where (i) the incident beam is parallel and point-like (having infinitesimally small cross section) and (ii) the scattered light is detected through an infinitesimally small pinhole. In the experiments, the scattered intensity distribution is smeared by (i) the divergence of incident beam, and finite cross sections of both (ii) the incident beam and (iii) a pinhole through which the scattered light is detected. The calculated intensity profile was therefore smeared according to the collimation system used in the experiment in a manner described in the Appendix and was compared with the experimental curves. The smeared calculated curves are shown by the solid curves in Figures 4 and 5 . All the curves shown in Figures 6 to 9 are the unsmeared calculated curves.

Comparison of Mie Scattering with R.G.B. Scattering. Approximated Methods to Evaluate Size and Refractive Index of the Fiber.

Figure 8 shows the effect of varying $m_{3}$ on the angular distribution of the calculated Mie scattering from fiber $\left(R_{1}=5.0 \mu \mathrm{m}\right.$ and $\left.m=1.548\right)$. The setting profile, when $m_{3}$ approaches $m$, is quite similar to that when $m$ approaches unity in Figure 7; (i) the scattered intensity rapidly decreases with increasing $\theta$ and (ii) the maxima

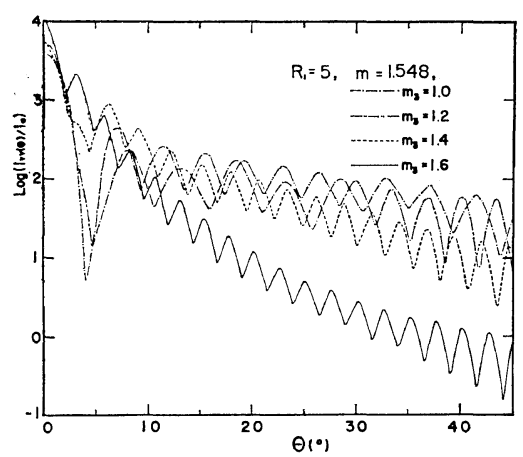

Figure 8. Calculated equatorial scattering curves for a cylinder of $R_{1}=5.0 \mu \mathrm{m}$ and $m=1.548$ as a function of $m_{3}$. shift toward the smaller angles, leading to decreased angular separation between the successive maxima owing to the same reason as discribed previously.

The effect of refraction of the scattered ray at the fiber-matrix interface may be partially corrected as follows. If $\theta_{\mathrm{s}}$ and $\theta$ are the scattering angles in the fiber and matrix, then

$$
\sin \theta_{\mathrm{s}}=\left(m_{3} / m\right) \sin \theta
$$

At small scattering angles

$$
\sin \left(\theta_{\mathrm{s}} / 2\right) \simeq\left(m_{3} / m\right) \sin (\theta / 2)
$$

If the reduce variable $U_{\mathrm{s}}$ is defined by

$$
U_{\mathrm{s}} \equiv\left(4 \pi / \lambda_{0}\right) m R_{1} \sin \left(\theta_{\mathrm{s}} / 2\right) \simeq\left(4 \pi / \lambda_{0}\right) m_{3} R_{1} \sin (\theta / 2)
$$

Therefore it is expected that the shift of the scattering maxima with refractive index $m_{3}$ (Figure 8 ) should be minimized by plotting the curves with $U_{\mathrm{s}}$ or $\theta_{\mathrm{s}}$ rather than $\theta$. The result is shown in Figure 9 where again $R_{1}=5.0 \mu \mathrm{m}$ and $m=1.548$. It is clearly seen that the shift of the scattering maxima with $m_{3}$ is small compared with that seen in Figure 8 . The residual shift of the scattering maxima with $m_{3}$ is a phenomenon inherent to the Mie scattering.

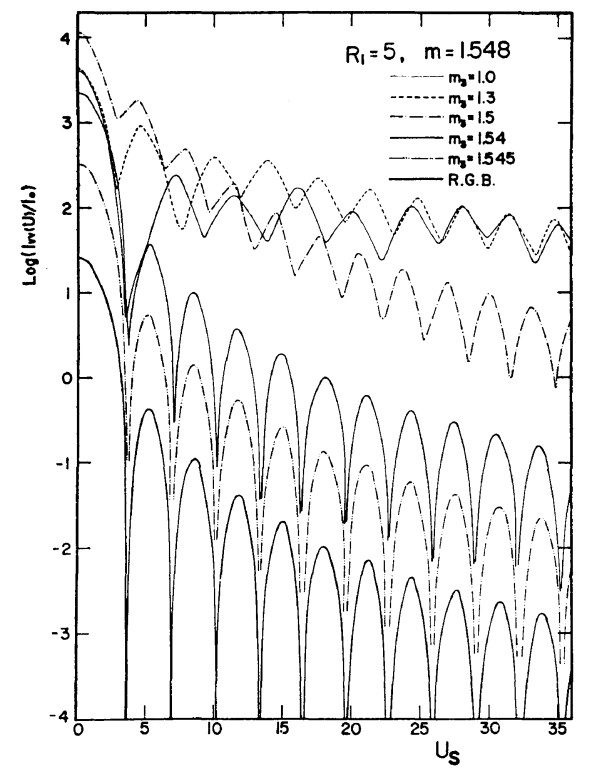

Figure 9. Same as Figure 8 but the relative intensity distribution is plotted as a function of reduced scattering angle, $U_{\mathrm{s}}=\left(4 \pi / \lambda_{0}\right) m R_{1} \sin \left(\theta_{\mathrm{s}} / 2\right)$ where $\theta_{\mathrm{s}}$ is the scattering angle in the fiber. 
Y. Uemura, M. Fujimura, T. Hashimoto, and H. Kawai

Table I. Estimated size of the glass fiber based upon eq 23

\begin{tabular}{|c|c|c|c|c|c|c|c|c|c|}
\hline & \multicolumn{3}{|c|}{$m_{3}=1.560$} & \multicolumn{3}{|c|}{$m_{3}=1.528$} & \multicolumn{3}{|c|}{$m_{3}=1.516$} \\
\hline & $i=6$ & $i=8$ & $i=10$ & $i=6$ & $i=8$ & $i=10$ & $i=6$ & $i=8$ & $i=10$ \\
\hline$\theta_{\max , i^{\mathrm{a}}}$ & 15.8 & 20.5 & 25.3 & 15.9 & 20.6 & 25.6 & 16.0 & 20.8 & 25.9 \\
\hline$R_{1}, \mu \mathrm{m}$ & 5.0 & 5.0 & 5.0 & 5.1 & 5.1 & 5.0 & 5.1 & 5.1 & 5.0 \\
\hline
\end{tabular}

a $U_{\max , i}=\left(4 \pi / \lambda_{0}\right) m_{3} R_{1} \sin \left(\theta_{\max , i} / 2\right)$

$=21.2,27.4$, and 33.7 for $i=6,8$, and 10, respectively.

Figure 9 also shows the R.G.B. scattering profile calculated on the basis of eq 8 and 9 where $U_{1}$ corresponds to $U_{\mathrm{s}}$. Mie scattering for the refractive indices $m_{3}=1.545$ and 1.540 are seen to be almost identical to the R.G.B. scattering. In these cases the criterion of eq 1 is satisfied as follows; $\left(4 \pi / \lambda_{0}\right) R_{1}\left|\left(m / m_{3}\right)-1\right|=0.192$ and 0.516 for $m_{3}=1.545$ and 1.540 , respectively. Upon a further increase of difference in the refractive indices $m$ and $m_{3}$, deviation from the R.G.B. scattering becomes larger. It should be noted, however, even for the scattering for $m_{3}=1.500$ (for which $\left(4 \pi / \lambda_{0}\right) R_{1}\left|\left(m / m_{3}\right)-1\right|=3.18$ and thus the situation is far from the criterion of the R.G.B. scattering, $\left.\left(4 \pi / \lambda_{0}\right) R_{1}\left|m / m_{3}-1\right| \ll 1\right)$ that (i) the relative intensity of the scattering maxima as a function of the orders of the maxima is very close to that found for the R.G.B. scattering, and that (ii) each peak position of the Mie scattering curve agrees with that of R.G.B. scattering within relative error of a few percents. Hence the R.G.B. theory turns out to be applicable with good accuracy even under a condition far from the R.G.B. criterion as long as interest is directed only to the peak positions and the relative height of the peaks. The intensity distribution of the R.G.B. scattering, however, quantitatively differs from that of the Mie scattering, especially around scattering minima when the criterion of the R.G.B. is not satisfied.

Based upon the discussions made above, we may propose an approximate method to estimate the size $R_{1}$ and the refractive index $m$ of the fiber. First we immerse the fiber in a liquid whose refractive index $m_{3}$ is close to that of the fiber. Then we measure the scattering angle $\theta_{\max , i}$ of the $i$ th scattering maximum. The radius $R_{1}$ of the fiber is then estimated from the scattering angle by using the following rela- tionship derived from the theoretical R.G.B. scattering curve,

$$
\left(4 \pi / \lambda_{0}\right) m_{3} R_{1} \sin \left(\theta_{\max , i} / 2\right)=U_{\max , i}
$$

where $\theta_{\max , i}$ is the scattering angle for the $i$-th scattering maximum. $U_{\max , i}$ is evaluated from the theoretical R.G.B. scattering curve shown at the bottom of Figure 9 and plotted in Figure 11 as a function of order $i$ of the scattering maximum. Accuracy of the estimated $\boldsymbol{R}_{1}$ depends on $\left|m-m_{3}\right|$; i.e., the smaller the value, the higher is accuracy.

Table I represents the estimated size of $R_{1}$ based upon eq 23 from the 6 th, 8 th, and 10 th scattering maxima of the fiber immersed in the oils of refractive indices of $1.516,1.528$, and 1.560. The scattering angles $\theta_{\max , i}(i=6,8$, and 10) of maximum intensity were measured from the curves as shown in Figure 5. The estimated results were found to agree with the literature value for the $E$-glass and with the value obtained

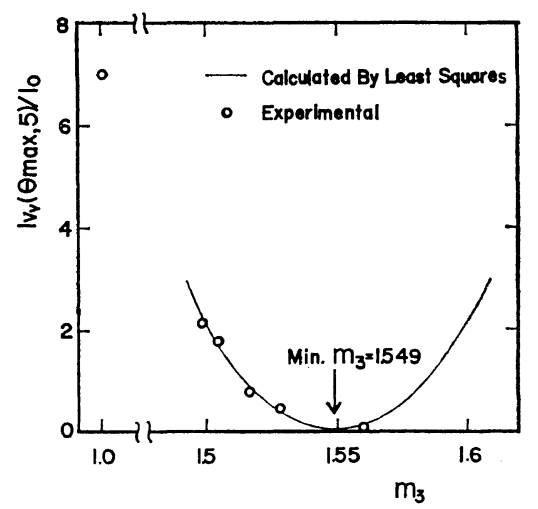

Figure 10. Plot of the fifth scattering maximum $I_{V_{\mathrm{V}}}\left(\theta_{\max , 5}\right)$ from the $E$-glass fiber as a function of refractive index of silicone oils (open circles). The solid line shows fit of the data points by the least squares with quadratic polynomial; $I_{V_{\nabla}}\left(\theta_{\max .5}\right)=$ 833.5 $\left(m_{3}-1.549\right)^{2}$. 
from a direct comparison of the theoretical Mie scattering and measured curves shown in Figure $4\left(R_{1}=5.0 \mu \mathrm{m}\right)$. Accuracy of the estimated size is, of course, higher for the measurements with $m_{3}$ closer to $m=1.548$.

The fractive index $m$ can be also evaluated by measuring the intensity of the $i$ th scattering maximum $I\left(\theta_{\max , i}\right)$ as a function of $m_{3}$ and by finding $m_{3}$ value which gives rise to the minimum scattering intensity, and these values should be equal to $m$. This procedure is represented in Figure 10 where the intensity of the fifth scattering maximum $I_{V_{\mathrm{v}}}\left(\theta_{\max , 5}\right)$ from the glass fiber was plotted as a function of refractive indices of silicone oils $m_{3}$. The fitting of the data-points with quadratic polynomial by the least squares (method) gave,

$$
I_{V_{\mathrm{V}}}\left(\theta_{\max , 5}\right)=833.5\left(m_{3}-1.549\right)^{2}
$$

This result has been indicated by the solid line in Figure 10. The estimated value of $m=1.549$ is close to 1.548 , the literature value of the $E$ glass. ${ }^{21}$ The maximum intensity parabolically varies with $m_{3}$ when the refractive index difference between the fiber and the surrounding medium is small, which indicates that the intensity variation is predicted by the R.G.B. scattering under this condition.

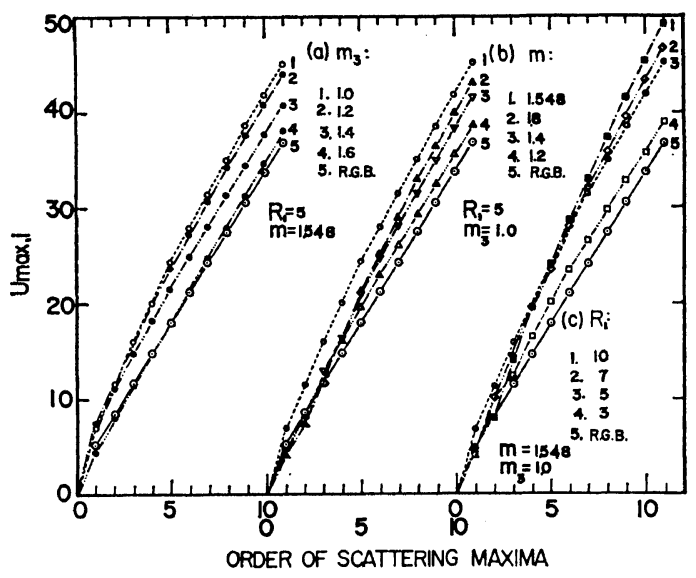

Figure 11. Plot of $U_{\max , i}$ as a function of the order $i$ of the scattering maxima for cases: (a) $R_{1}=5.0 \mu \mathrm{m}$ and $m=1.548$ for various values of $m_{3}$; (b) $R_{1}=5.0 \mu \mathrm{m}$ and $m_{3}=1.0$ for various values of $m$; (c) $m=1.548$ and $m_{3}=1.0$ for various value of $R_{1}$.
Figure 11 represents the plot of $U_{\max , i}$, the reduced scattering angle $U_{\mathrm{s}}$ for the $i$ th scattering maximum, as a function of the order $i$ of the scattering maxima for the cases: (a) $R_{1}=5.0 \mu \mathrm{m}$ and $m=1.548$ for various values of $m_{3}$; (b) $R_{1}=$ $5.0 \mu \mathrm{m}$ and $m_{3}=1.0$ for various values of $m$; (c) $m=1.548$ and $m_{3}=1.0$ for various values of $R_{1}$. It is seen from the figure that the slope of $U_{\max , i}$ against $i$ of the Mie scattering generally approaches to a constant value equal to that of the R.G.B. scattering for the value of $i$ or $U_{\max , i}$ which is greater than a critical value. The critical value depends on the difference of the refractive indices between the fiber and the medium as in Figures $11(\mathrm{a})$ and $11(\mathrm{~b})$, i.e., the smaller the difference in the refractive indices, the smaller the critical value. For an example, for $m_{3}=1.4$ in Figure 11(a), the critical value for $U_{\max , i}$ is about 10 and that for $i$ is about 2. The critical value also depends upon the size of the fiber as in Figure 11(c), i.e., the smaller the size, the smaller is the critical value. This implies that the angular separation of $\Delta U_{\max , i}$ between the $i$ th and $(i-1)$ th scattering maxima $\left(\Delta U_{\max , i}=U_{\max , i}-U_{\max ,(i-1)}\right)$ becomes a constant equal to that expected for the R.G.B. scattering.

Based upon the discussions above, one may propose an approximate method to evaluate $R_{1}$ from the measured and calculated $\Delta U_{\max , i}$ :

$$
\begin{aligned}
\Delta U_{\max , i}= & U_{\max , i}-U_{\max ,(i-1)} \\
= & \left(4 \pi / \lambda_{0}\right) m_{3} R_{1}\left[\sin \left(\theta_{\max , i} / 2\right)\right. \\
& \left.-\sin \left(\theta_{\max , i-1} / 2\right)\right]
\end{aligned}
$$

where

$$
\Delta U_{\max , i}=3.14 \text { for } i \gtrsim 7
$$

The larger the fiber size and the greater the difference in the refractive indices of the fiber and the surrounding medium, the larger the critical value of $i$.

Table II represents the estimated size of $R_{1}$ of the fiber based upon eq 24 and 25 . The estimation utilizes the scattering angles of the eighth and nineth scattering maxima for the fiber immersed in media of various refractive indices. These values are comparable with those estimated by direct comparison of the calculated and measured scattering curves (Figure 4) and by the R.G.B. approximation for the immersed 
Table II. Estimated size of the glass fiber based upon eq 24 and 25

\begin{tabular}{|c|c|c|c|c|}
\hline \multirow{2}{*}{$\begin{array}{l}\text { Value } \\
\text { of } m_{3}\end{array}$} & \multicolumn{2}{|c|}{$\begin{array}{l}\text { Experimentally } \\
\text { detected }^{\mathrm{a}}\end{array}$} & \multirow{2}{*}{$\begin{array}{l}\text { Radius of } \\
\text { the fiber } \\
R_{1}, \mu \mathrm{m}\end{array}$} & \multirow{2}{*}{ Remarks } \\
\hline & $\theta_{\max , i}$ & $\theta_{\max , i-1}$ & & \\
\hline 1.000 & 45.0 & 41.2 & 5.1 & Figure 4 \\
\hline 1.498 & 23.1 & 20.7 & 5.1 & \\
\hline 1.504 & 23.0 & 20.5 & 4.9 & \\
\hline 1.516 & 23.1 & 20.7 & $5.1\}$ & Figure 5 \\
\hline 1.528 & 23.0 & 20.6 & 5.0 & \\
\hline 1.560 & 22.7 & 20.3 & 4.9) & \\
\hline
\end{tabular}

a For $i=9$ in degrees.

specimens (eq 23). It should be noted that eq 25 can be applied, in a more general fashion than eq 23 , even to systems with values of $m_{3}$ far from the R.G.B. criterion.

\section{CONCLUSION}

It turns out that the light scattering method is useful as a means for a rigorous estimation of the size and refractive index of glass fiber. This technique may be especially useful when applied to a fiber whose size is too small for observation under an optical microscope. The size and refractive index were evaluated by direct comparison of the experimental and calculated Mie scattering intensity distributions in a forward direction and by analyzing the measured intensity distribution from the fiber immersed in silicone oils in terms of R.G.B. and Mie scattering. These results agreed with the literature values of the $E$-glass fiber.

\section{APPENDIX}

The Smearing of the Scattering Intensity Distribution Due to Finite Angular Resolution of the Apparatus

Let the intensity distribution of the incident beam at the detector plane be $i_{0}\left(u_{1}, u_{2}\right)$. If $I$ and $I_{\text {obs }}$ are the true intensity and observed smeared intensity, respectively, then the observed intensity at a reduced scattering angle $h$ (see Figure 12 ) is given by

$$
I_{\mathrm{obs}}(h)=\frac{\iint i_{0}\left(u_{1}, u_{2}\right) I\left(h-u_{1}, u_{2}\right) \mathrm{d} u_{1} \mathrm{~d} u_{2}}{\iint i_{0}\left(u_{1}, u_{2}\right) \mathrm{d} u_{1} \mathrm{~d} u_{2}}
$$

where $h=(4 \pi / \lambda) \sin (\theta / 2)$ and $u_{i}=(4 \pi / \lambda) \sin \left(\theta_{i} / 2\right)$ $(i=1$ and 2$)$

If the scattering from an infinitely long glass fiber oriented parallel along the $z$-axis is to be considered, it obviously follows that

$$
I\left(h-u_{1}, u_{2}\right)=I\left(h-u_{1}\right) \delta\left(u_{2}\right)
$$

where $\delta$ is Dirac's delta function. Then

$I_{\mathrm{obs}}(h)=\int i_{0}\left(u_{1}, 0\right) I\left(h-u_{1}\right) \mathrm{d} u_{1} / \int i_{0}\left(u_{1}, 0\right) \mathrm{d} u_{1}$,

where $I\left(h-u_{1}\right)$ is the calculated equatorial Mie scattering and $i_{0}\left(u_{1}, 0\right)$ is the measured intensity profile of the incident beam along the horizontal plane. In the experimental system used in this work, the detecting optical system has a circular aperture of $1.4 \mathrm{~mm}$ in diameter and the spread of laser beam at the aperture is $1 \mathrm{~mm}$ in diameter. The distance between the sample and slit position is $80 \mathrm{~mm}$. In this optical system, the profile of $i_{0}\left(u_{1}, 0\right)$ was obtained as in Figure 13. The smeared curves in the paper were calculated by using the weighting function $i_{0}\left(u_{1}, 0\right)$.

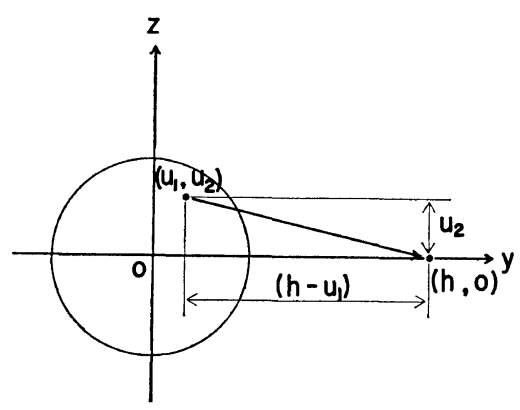

Figure 12. Calculation of the smearing of intensity distribution due to imperfect collimation of the optical system.

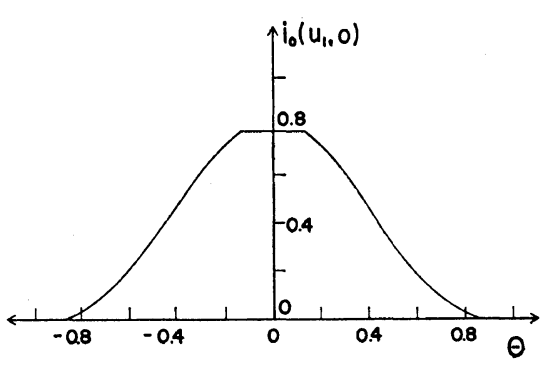

Figure 13. Estimated weighting function to calculate the effect of smearing.

Polymer J., Vol. 10, No. 3, 1978 


\section{REFERENCES}

1. L. Rayleigh, Proc. Roy. Soc., 84, A25 (1911); 90, 219 (1914).

2. R. Gans, Ann. Physik, 76, 29 (1925).

3. M. Born, Z. Phys., 37, 863 (1926).

4. H.C. Van de Hulst, "Light Scattering by Small Particles," John Wiley \& Sons, Inc., New York, N.Y., 1957.

5. See for example, J. C. Slater and N. H. Frank, "Electromagnetism," McGraw-Hill, New York, N.Y., and London, 1947, Chapter 12; J. A. Stratton, "Electromagnetic Theory," McGrawHill, New York, N.Y., 1941, Chapter VIII.

6. See for example, "Light Scattering from Dilute Polymer Solutions," D. McIntyre and F. Gornick, Ed., Gordon and Breach, New York, N.Y., 1964.

7. See for example, B. Chu, "Molecular Forces; Based on the Baker Lectures of Peter J.W. Debye," John Wiley \& Sons Inc., New York, N.Y. 1967; R. S. Stein, in "Structure and Properties of Polymer Films," R. W. Lenz and R. S. Stein, ed, Plenum Press, New York, N.Y., 1973.

8. V. K. La Mer and M. D. Barnco, J. Colloid Sci., 1, 71 (1946).

9. W. Heller and M. L. Wallach, J. Phys. Chem. 68, 931 (1964).

10. M. Kerker, "Scattering of Light and Other
Eletromagnetic Radiation," Academic Press, New York, N.Y., 1969.

11. G. Mie, Ann. Phys., 29, 37 (1908).

12. S. W. Churchill, J. Opt. Soc. Am., 49, 188 (1959).

13. D. D. Cooke and M. Kerker, J. Opt. Soc. Am., 59, 43 (1969).

14. W. A. Farone and R. W. Queenfield, J. Opt. Soc. Am., 56, 476 (1966).

15. W. A. Farone and M. Kerker, J. Opt. Soc. Am., 56, 481 (1966).

16. J. L. Lundberg, J. Colloid and Interface Sci., 29, 565 (1969).

17. C. C. Tang, J. Appl. Phys., 8, 628 (1957).

18. L. B. Evans, J. C. Chen, and S. W. Churchill, J. Opt. Soc. Am., 54, 1004 (1964).

19. M. Kerker and E. Matijerrc, J. Opt. Soc. Am., 51, 506 (1961).

20. C. Yeh, J. Opt. Soc. Am., 54, 1227 (1964).

21. E. B. Shand, "Glass Eng. Handbook,"' 2nd ed, McGraw-Hill, New York, N.Y., 1958, p 325.

22. I. A. Stegum and M. Abramorvitz, Math. Tab. and Other Aids to Computation, 11, 255 (1957).

23. Y. Uemura, T. Hashimoto, and H. Kawai, to be submitted to Polym. $J$.

24. For numerical calculation, the Hankel function of the second kind of order $n, H_{n}(x)$, was expressed in terms of $J_{n}(x)$ and $Y_{n}(x)$, each of which was numerically calculated as described in the text. $H_{n}(x)=J_{n}(x)-i Y_{n}(x)$. 